\title{
Fabrication of silver nanowires via a $\beta$-cyclodextrin-derived soft template
}

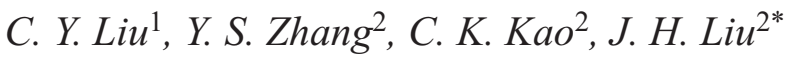 \\ ${ }^{1}$ School of Optometry and Vision Science Program, UC Berkeley, CA 94720 Berkeley, USA \\ ${ }^{2}$ Department of Chemical Engineering, National Cheng Kung University, 70101 Tainan, Taiwan (ROC)
}

Received 4 December 2017; accepted in revised form 30 January 2018

\begin{abstract}
Supramolecular $\beta$-cyclodextrin $(\beta-C D)$ was used as a soft template for the fabrication of long silver nanowires. A novel design using self-assembled $\beta-C D$ for the reduction of silver ions was studied. The concentrations of iron chloride, silver nitrate, and the template were controlling factors for the growth of the silver nanowires. Iron chloride was used to accelerate and facilitate the formation of the silver nanowires and inhibit oxidative etching. However, an excessive concentration of $\mathrm{Fe}^{+3}$ resulted in etching of the silver nanostructures. Furthermore, the silver concentration was another controlling factor. The length of the silver nanowires increased as the concentration of silver cations increased. Nevertheless, an excess concentration of silver cations formed various silver crystalline structures. In this study, the optimal ratio between iron chloride and silver nitrate was determined to be 1:13.3. A maximum length of $20 \mu \mathrm{m}$ was achieved using a concentration of $0.23 \mathrm{M}$ for the soft template. Moreover, the junction of two growing silver nanowires was observed, forming a long fused nanowire, and some significant boundaries were observed. The observed results were further confirmed using scanning electron microscopy (SEM) and transmission electron microscopy (TEM) analyses. X-ray diffraction (XRD) and energy dispersive spectrometer (EDS) analyses were used to indicate the presence of silver and the formation of crystalline materials.
\end{abstract}

Keywords: nanomaterials, $\beta$-cyclodextrin, heat-induced sol-gel template, silver nanowires

\section{Introduction}

Silver is one of the most important industrial metals in modern technologies, with the highest electrical and thermal conductivity of any metal and the lowest contact resistance, which has allowed it to be used as an interconnection, particularly in the form of wires $[1,2]$, in a wide variety of commercial applications, including conductive inks, electronic products, cosmetics, antibacterial medical products and coatings [3].

Currently, nanotechnology is aimed at fabricating functional and fine-tuned materials, devices and systems through the control of matter at the nanometerlength scale of less than $100 \mathrm{~nm}$, where properties differ significantly from those at larger scales. For example, although both bulky silver and nanosilver possess high thermal and electrical conductivities, bulky silver has a metallic color and a high melting point (above $900^{\circ} \mathrm{C}$ ), which diverges from the properties of nanosilver (i.e., light-yellow color in solution and antibacterial properties). These differences lead to 'nano-generation'. Therefore, silver nanoparticles have emerged as one of the fastest growing material categories in the nanotechnology industry. It is very attractive, based on both fundamental and technological aspects, to fabricate the size, shape, and assembly of the inorganic nanoparticles because they play important roles in many areas, such as electronic, magnetic, optical, optoelectronic, and catalytic fields [4-11]. The size and shape of organic compounds can be controlled using building blocks for constructing an extended solid-state structure with 
predesigned properties [12-14]. Because of their unique properties and applications, silver nanoparticles have attracted great interest in our daily life [1520]. Accordingly, methods must be designed to prevent the formation of large metal clusters in their growth processes. Template methods using a variety of materials, such as alumina membranes, porous polycarbonates, carbon nanotubes, DNA chains, rodlike micelles, and organogels, have been employed in the growth of silver nanowires [21-30].

Organogels, particularly stimuli-responsive gels, have been extensively investigated and have potential applications in smart biomaterials and drug-delivery systems [31-33]. The gelation of small organic molecules usually occurs by the formation of fibrils and self-assembled networks based on supramolecular interactions [34].

Our group is interested in utilizing the properties of these gels to construct high-order templates to synthesize silver nanowires. Nguyen and Liu [35] fabricated silver nanowires based on a soft template of a cholesteryl pyridine carbamate organogel. For their synthesis, silver nanowires must be under high-temperature conditions; however, gels usually form in ambient environments. In the case of the polyol synthesis method, which is the most common method for synthesizing silver nanowires, the number of functional groups must be limited to inhibit or promote the formation of specific crystalline facets leading to wires. Therefore, to overcome the above limitations, we introduce reversible heat-set gels, which are usually prepared from small organic molecules. These reversible heat-set organogels would be very valuable in thermo-responsive and self-assembling materials and other fields. As important supramolecular hosts, skirt-shaped cyclodextrins (CDs, including $\alpha-, \beta-$, and $\gamma$-CD) with hydrophobic pockets and hydrophilic exteriors can form complexes with various organic guest molecules through supramolecular interactions [36-40]. To the best of our knowledge, no heat-set $\mathrm{CD}$ organogel has been reported; however, many gels based on CDs have been prepared. These findings of a heat-set organic CD gel could open a door for the design of new low-cost and efficient multicomponent organogelators and would be very valuable in the delivery of functional molecules and in the design of intelligent materials, biomaterials, and other systems. Herein, we report a heat-set organogel based on $\beta$-cyclodextrin $(\beta-C D)$. This gel system is a thermal responsive organogel of small organic molecules formed by a system of CD and $\mathrm{LiCl}$ in DMF.

\section{Experimental}

\subsection{Chemicals}

$\beta$-Cyclodextrin ( $\beta$-CD, $97 \%)$, silver nitrate $\left(\mathrm{AgNO}_{3}\right.$, extra pure), lithium chloride ( $\mathrm{LiCl}, 99 \%), \mathrm{N}, \mathrm{N}$-dimethylformamide (DMF, 99.8\%), and iron(III) chloride $\left(\mathrm{FeCl}_{3}, 97 \%\right.$, anhydrous) were purchased from Tokyo Chemical Industry (TCI), Tokyo, Japan. All chemical reagents were of analytical grade.

\subsection{Measurements}

Scanning electron microscopy (SEM) investigations were carried out using a JEOL HR-FRSEM JSM6700F and a Hitachi HR-FESEM SU-8010.

The SEM samples were prepared on clean glass substrates. The samples were then coated with Pt by ion sputtering (Hitachi, $\varepsilon-1010)$. Transmission electron microscopy (TEM) analyses were carried out on a Hitachi HF-7500 Field Emission Transmission Election Microscope. The TEM samples were prepared by drop coating onto a 200-Mech carbon-coated copper grid followed by solvent evaporation in a vacuum oven overnight. X-ray diffraction (XRD) data were recorded with a D/Max-2500/PC powder diffractometer (Rigaku, Japan) operated at $40 \mathrm{kV}$ and $40 \mathrm{~mA}$ using Ni-filtered $\mathrm{CuK} \alpha$ (wavelength $=1.54 \AA$ ) radiation.

\subsection{Synthesis of silver nanowires}

The heat-induced reversible gel was prepared using the procedure reported by Li et al. [41] and described as follows. A pre-determined concentration of $\beta-C D$ and $0.5 \mathrm{wt} \% \mathrm{LiCl}$ in DMF were added to a $50 \mathrm{~mL}$ double-neck bottle setup with a stirrer and condenser and heated to $170^{\circ} \mathrm{C}$ in a sand bath, resulting in a white-colored gel. A solution of $\mathrm{FeCl}_{3}$ in DMF was introduced dropwise to the obtained gel. During the addition of $\mathrm{FeCl}_{3}$, the color of the gel changed from white to light yellow, and the gel transitioned from the rigid state to the partial gel state. Silver nitrate was dissolved in DMF, and the resulting mixture was sonicated for 10 minutes [42]. The $\beta$-CD system was held at $170^{\circ} \mathrm{C}$ for 10 minutes and then silver nitrate was then injected dropwise into the twoneck bottle. The color sequentially changed from light yellow to milky white, dark gray and reddish brown. The mixture was heated for 1 hour and then was allowed to rest at room temperature overnight $(>12 \mathrm{~h})$. 
The synthesized silver nanowires contained many impurities, such as $\mathrm{AgCl}, \beta-\mathrm{CD}$ and other cations. The crude product was washed with water, $1 \mathrm{M}$ aqueous ammonia and then ethanol three times via a centrifuge. The unreactive $\beta-C D$ and ions were removed thoroughly by washing, and $\mathrm{AgCl}$ were removed according to the following reaction [43]:

$$
\mathrm{AgCl}+\mathrm{NH}_{4} \mathrm{OH} \leftrightarrows \mathrm{Ag}\left(\mathrm{NH}_{3}\right)_{2}^{+}+\mathrm{Cl}^{-}
$$

Ethanol was used to remove aqueous ammonia and promote the dispersion of the samples for SEM and TEM analyses.

\section{Results and discussion}

$\beta$-Cyclodextrin ( $\beta$-CD) can form an organogel at elevated temperatures with $N, N$-dimethylformamide (DMF) containing a small amount of lithium chloride. Figure 1 shows a photograph of the heatinduced $\beta-C D$ organogel. The transparent solution of the $\beta$ $\mathrm{CD}$ system formed an opaque gel when heated, and the gel reversibly transformed into a solution when cooled to room temperature. This result is ascribed to the self-assembly of cyclodextrin under the specified conditions when heated. The concentration of $\beta-C D$ in DMF affected the gelation temperature (Tgel), which decreased as the concentration of $\beta$ $\mathrm{CD}$ increased. The range of the permitted $\beta-\mathrm{CD}$ concentration was estimated to be 0.13 to $0.28 \mathrm{M}$. Within this range, heat-induced reversible sol-gel transition was observed. A concentration of $0.5 \mathrm{wt} \% \mathrm{LiCl}$ in DMF was calculated to be the key factor for the gelation system. A schematic representation of the formation of the gel via self-assembly is shown in Figure 2. As shown in the figure, $\mathrm{LiCl}$ plays a significant
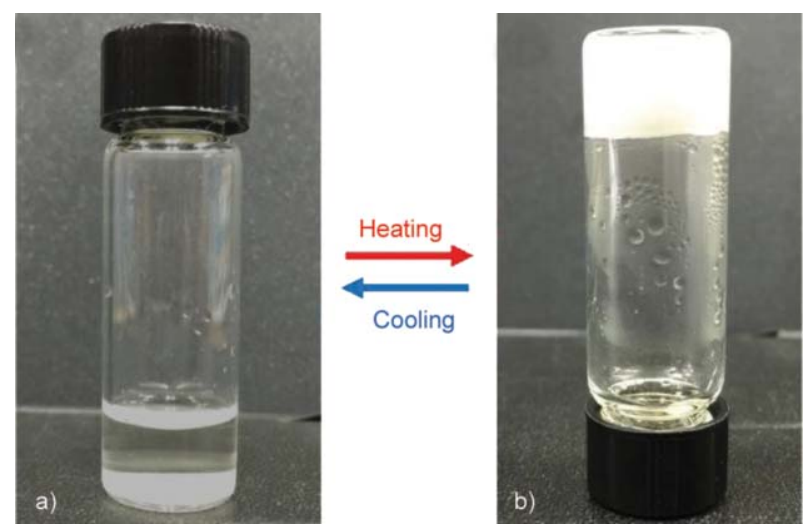

Figure 1. Photographs of sol-gel phase transition of the $\beta-\mathrm{CD} / \mathrm{DMF} / \mathrm{LiCl}$ system: (a) a transparent solution at room temperature and (b) white gel phase formed at elevated temperatures.

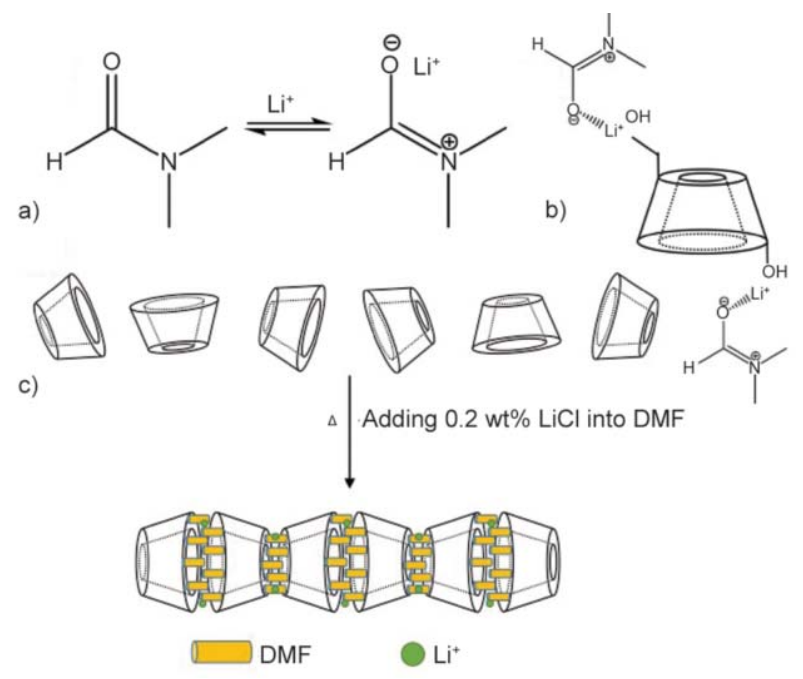

Figure 2. (a) Emergence of the $\mathrm{C}=\mathrm{N}$ bond from $\mathrm{DMF}$ influenced by $\mathrm{Li}^{+}$; (b) the polar-polar interaction between $\mathrm{LiCl} / \mathrm{DMF}$ and $\beta-\mathrm{CD}$; (c) schematic illustration of the self-assembly process.

role in the formation of the gel. In the absence of $\mathrm{LiCl}$, the system formed a precipitate in DMF. Moreover, $\mathrm{LiCl}$ supplied chloride anions, which reacted with silver to form silver chloride, serving as a reaction rate controller during the silver nanowire synthesis. As shown in Figure 2 and Figure 3d silver ions may interact with the polar groups and ionic sites on the complexes. The $\beta$-CD gels showed selfassembled linear structures, as shown in Figure 3a. Thus, the obtained self-assembled linear structures are expected to act as a soft temple for the fabrication of silver nanowires. The addition of silver ions into the system, as shown in Figure $3 \mathrm{~b}$ and $3 \mathrm{c}$ resulted in the fabrication of some reduced silver nanoparticles. The results suggest that some of the silver ions interacted with the soft template leading to the formation of silver particles. From the fabrication process shown in Figure 2, it can be predicted that the concentrations of silver nitrate and the template as well as the molar ratio of iron chloride to lithium chloride might affect the formation and growth of the silver nanowires.

To successfully fabricate the silver nanowires, the silver nitrate concentration was optimized using a series of $\mathrm{AgNO}_{3}$ concentrations. The morphologygies of the fabricated samples and the growth of the silver nanowires were investigated using SEM and TEM. The detailed fabrication conditions and the fabricated wire lengths are summarized in Table 1. The SEM images of the fabricated products are shown in Figure 4. For sample S1, only a limited amount of silver nitrate was added. As shown in Figure 4a to 4c 


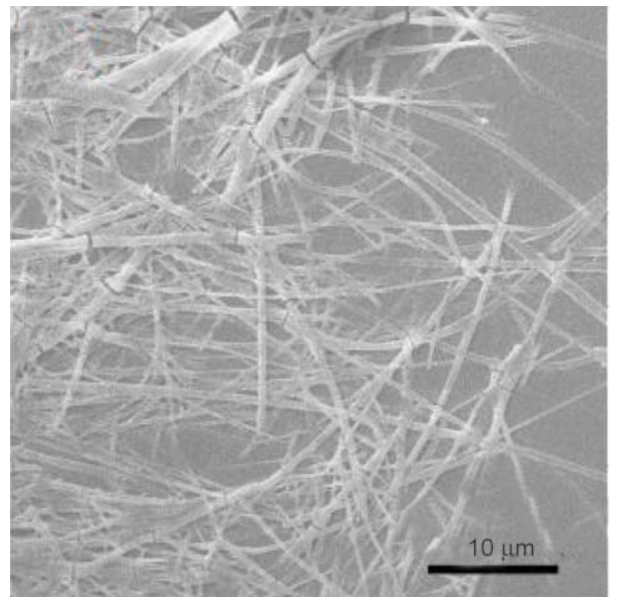

a)

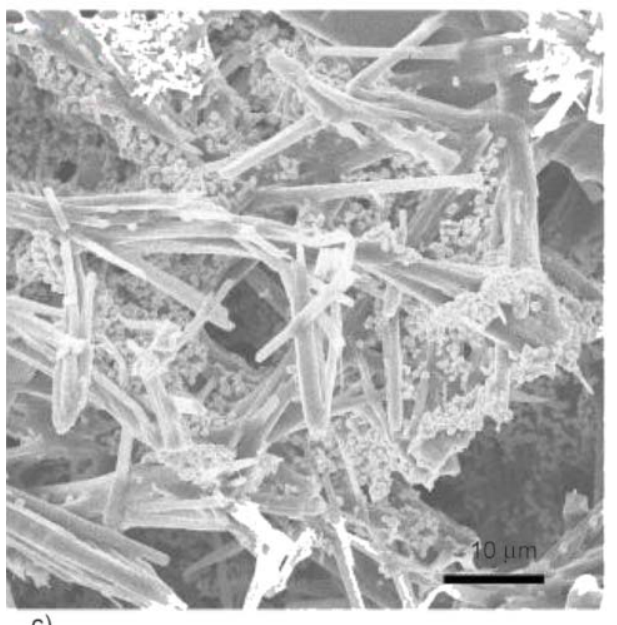

c)

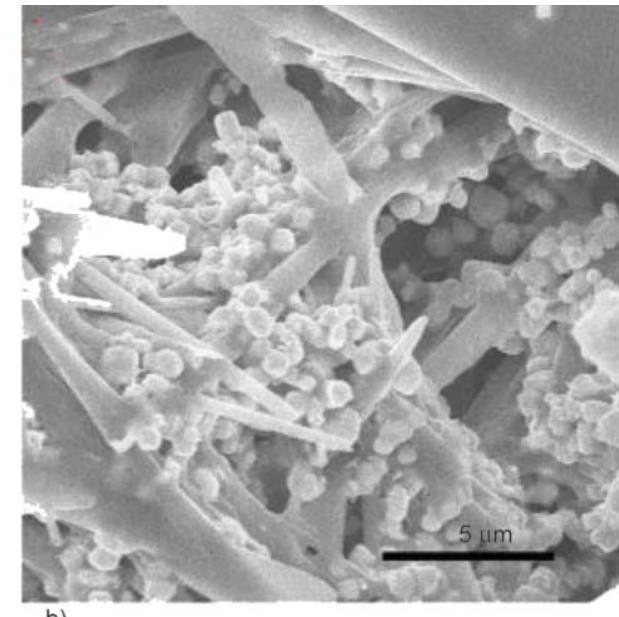

b)
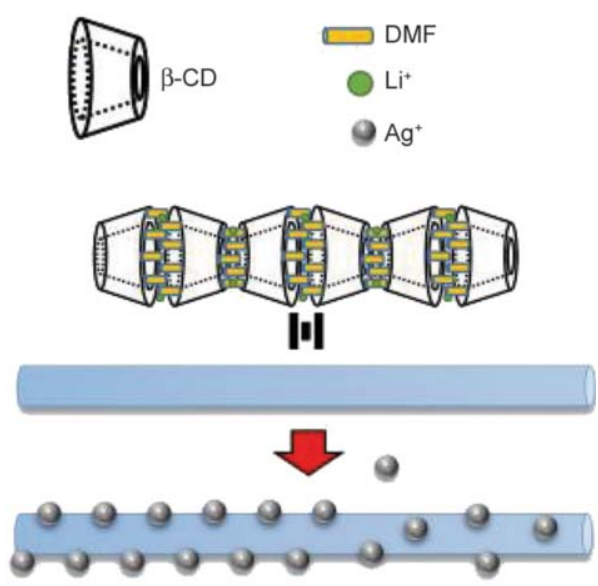

d)

Figure 3. (a) SEM image of the fabricated xerogel $(C \beta-C D=0.16 \mathrm{M}$ with $0.5 \mathrm{wt} \% \mathrm{LiCl})$; (b) and (c) SEM images of the reduced silver nanowires and nanoparticles via the $\beta-\mathrm{CD}$ template at different magnifications $(\mathrm{C} \beta-\mathrm{CD}=0.16 \mathrm{M}$ with $\mathrm{LiCl}: \mathrm{AgNO}_{3}=1: 3$ without $\mathrm{FeCl}_{3}$ ); (d) schematic representation of the fabrication of silver nanowires via the heatinduced soft template.

Table 1. Effect of silver nitrate concentration ${ }^{\mathrm{a}}$.

\begin{tabular}{|c|c|c|c|}
\hline Sample & $\mathbf{L i C l}_{\mathbf{F e C l}}: \mathbf{A g N O}_{\mathbf{3}}$ & $\mathbf{C l}^{-}: \mathbf{A g}^{+\mathbf{b}}$ & Max length $^{\mathbf{c}}$ \\
\hline S1 & $3: 1: 2.4$ & $1: 0.4$ & - \\
\hline S2 & $3: 1: 6.6$ & $1: 1.1$ & 4 \\
\hline S3 & $3: 1: 10$ & $1: 1.7$ & 5 \\
\hline S4 & $3: 1: 13.3$ & $1: 2.2$ & 16 \\
\hline S5 & $3: 1: 20$ & $1: 3.3$ & 11 \\
\hline
\end{tabular}

${ }^{\mathrm{a}}[\beta-\mathrm{CD}]=0.13 \mathrm{M}$ in DMF at $170^{\circ} \mathrm{C} .{ }^{\mathrm{b}} \mathrm{Calculated}$ ion molar ratio. ${ }^{\mathrm{c} F a b r i c a t e d ~ s i l v e r ~ n a n o w i r e ~}[\mu \mathrm{m}]$.

instead of silver nanowires, some silver chloride precipitates were observed, which was further confirmed by XRD analysis. Moreover, the precipitated $\mathrm{AgCl}$ exhibited cubic and octahedral morphologies under SEM and TEM analyses, as shown in Figure $4 \mathrm{a}$ to $4 \mathrm{c}$. As the concentration of $\mathrm{AgNO}_{3}$ increased, the nanowires gradually became longer, reaching a maximum length of $16 \mu \mathrm{m}$ for sample $\mathrm{S} 4$, as shown in Figure 4h. The fabricated silver wires were further confirmed using TEM, as shown in Figure $4 \mathrm{e}$ to $4 \mathrm{~h}$. It has been well documented that slow reduction during the initial growth stages facilitates the formation of pentagonal twinned particles, which can rapidly grow into silver nanowires when a sufficient amount of silver source is supplied (sample S2 to S5). In this experiment, the first slow dropwise addition of silver nitrate resulted in the precipitation of silver chloride. Dynamic release of silver from silver chloride acted to control the reduction rate. However, the successive addition of silver nitrate provided a sufficient amount of silver source for fast anisotropic growth. Finally, silver wires were fabricated via the reduction process.

The XRD analysis of the fabricated samples is shown in Figure 5. The reflection peaks greater than $30^{\circ}$ were indexed as the (111), (200), (220), and (311) planes of face-centered cubic (FCC) silver (JCPDS card No. 04-0783). The XRD analysis results indicate 


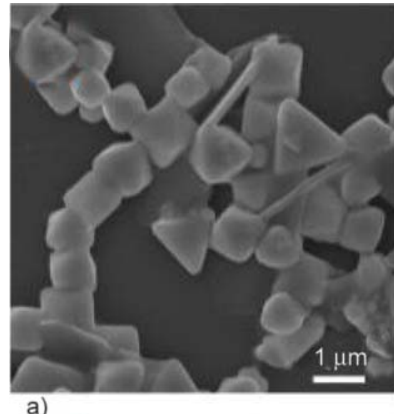

a)

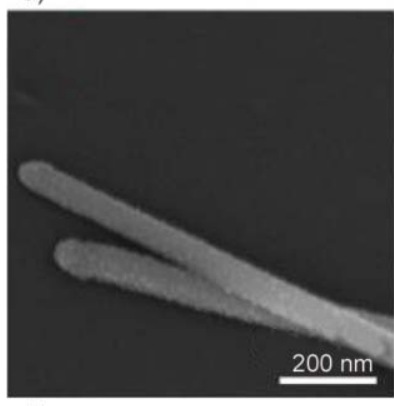

d)
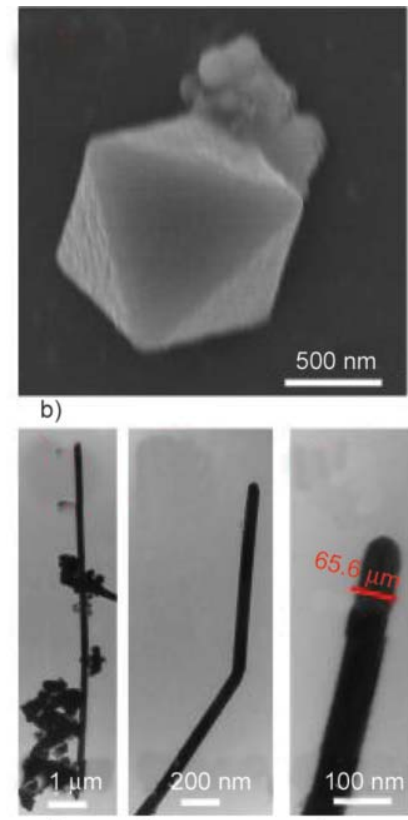

e)

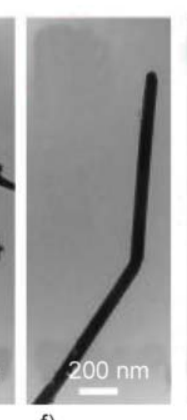

f)

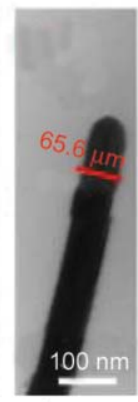

g)

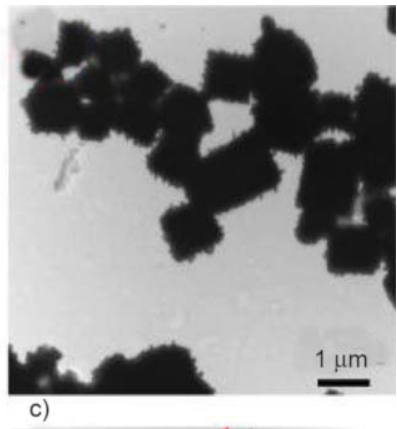

c)

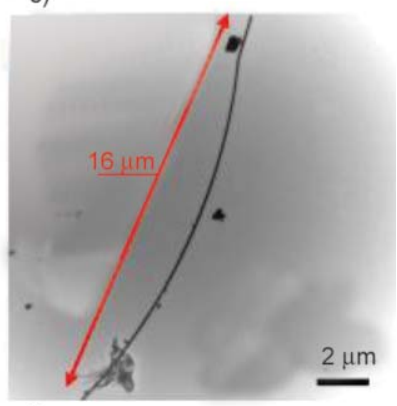

h)

Figure 4. (a) and (b) SEM images and (c) TEM image of S1 showing that AgCl precipitates formed; (d) SEM image and (e) and (f) TEM images of S4 showing that the product is mainly composed of Ag nanowires; ( $g$ ) and (h) TEM images confirming the maximum width and length of the $\mathrm{Ag}$ nanowires.

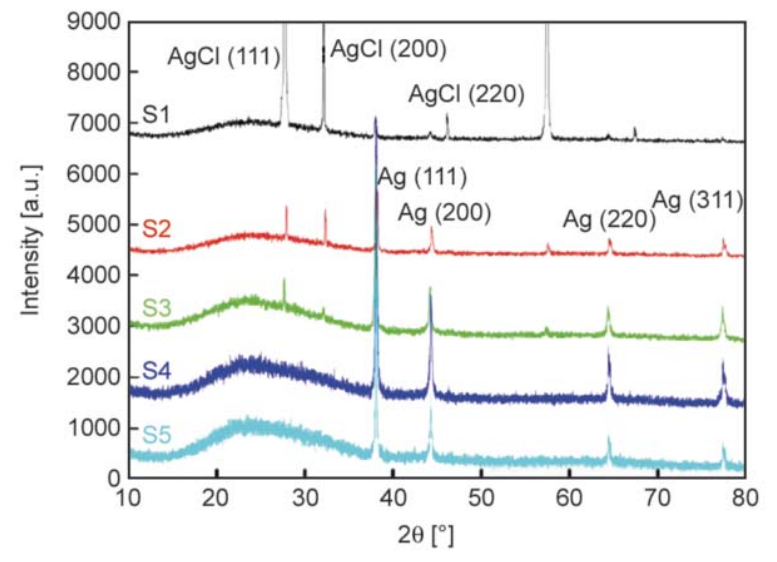

Figure 5. XRD analysis of the fabricated samples with different silver nitrate concentrations.

that the fabricated amount of silver and the crystallinity of silver increased as the silver nitrate concentration increased.

Furthermore, the intensity ratio between the (111) and (200) peaks in sample S4 was 2.14 (the theoretical ratio is 2.5), suggesting a deficiency of (111) crystalline planes in the silver nanowires. The silver chloride reflection peaks are marked in Figure 5 (JCPDS card No. 31-1238), which decreased upon raising the amount of silver additive in the system. Energy-dispersive X-ray spectroscopy (EDS) was used for further analyze sample S4.

The atomic percentage of $\mathrm{Ag}$ was $77.05 \%$ in the sample, as confirmed by Figure 6 .

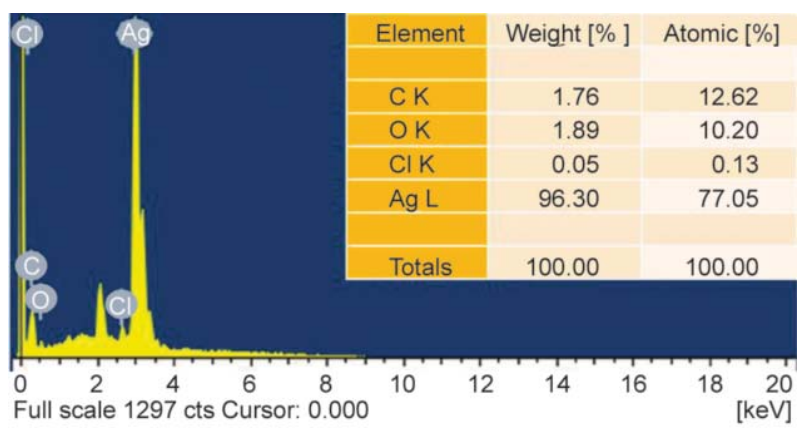

Figure 6. EDS analysis of the silver nanowires in S4.

It was found that the control agent, iron chloride, played a significant role in the production of the silver nanowires [44]. The necessity for iron chloride $\left(\mathrm{FeCl}_{3}\right)$ was based on the following traits:

(i) Chloride anions could be used to control the reaction rate in a similar fashion as $\mathrm{LiCl}$.

(ii) $\mathrm{Fe}^{+3}$ cations could prevent oxidative etching of the silver nanowires. DMF can reduce $\mathrm{Fe}^{+3}$ to $\mathrm{Fe}^{+2}$, and $\mathrm{Fe}^{+2}$ can rapidly scavenge oxygen, leading to the oxidation of $\mathrm{Fe}^{+2}$ to $\mathrm{Fe}^{+3}$.

To determine the effects of iron chloride on the fabrication of silver nanowires, as shown in Table 2, a series of iron chloride concentrations was used. The results of the fabricated silver wires are summarized in Table 2. Increasing the iron chloride concentration from 0.5 to $1.0 \mathrm{M}$ resulted in the formation of silver nanowires. However, when the iron chloride concentration was increased to twice that of sample F2, 
Table 2. Effect of iron chloride concentration ${ }^{\mathrm{a}}$.

\begin{tabular}{|l|c|c|c|}
\hline \multicolumn{1}{|c|}{ Sample } & $\mathbf{L i C l}_{\mathbf{F e C l}}$ & $\mathbf{C l}^{-}: \mathbf{A g}^{+}$ & Max. length \\
\hline F1 & $3: 0.5$ & $1: 3.0$ & - \\
\hline F2(S4) & $3: 1.0$ & $1: 2.2$ & 16 \\
\hline F3 & $3: 2.0$ & $1: 1.5$ & 4 \\
\hline F4 & $3: 3.0$ & $1: 1.1$ & 4 \\
\hline
\end{tabular}

${ }^{\mathrm{a}}[\beta-\mathrm{CD}]=0.13 \mathrm{M}$ in $\mathrm{DMF}$ at $170^{\circ} \mathrm{C}$ with fixe amounts of silver nitrate and lithium chloride. Molar ratio was used.

along with the formation of silver nanowires, silver nanoparticles were etched by $\mathrm{Fe}^{+3}$ (Figure 7c). In addition, the maximum wire length was reduced to $4 \mu \mathrm{m}$ for samples $\mathrm{F} 3$ and $\mathrm{F} 4$. When the ratio of $\mathrm{FeCl}_{3}$ was equimolar to $\mathrm{LiCl}$, too many iron cations were present, which destroyed the $\beta$-CD template, leading to the formation of several kinds of silver nanowire morphologies (Figure $7 \mathrm{~b}$ and $7 \mathrm{~d}$ ). The average diameters of the fabricated silver nanowires in F2-F4 were approximately $50 \sim 100 \mathrm{~nm}$.

Notably, the formation of silver nanoparticles was only observed when iron chloride was not present in

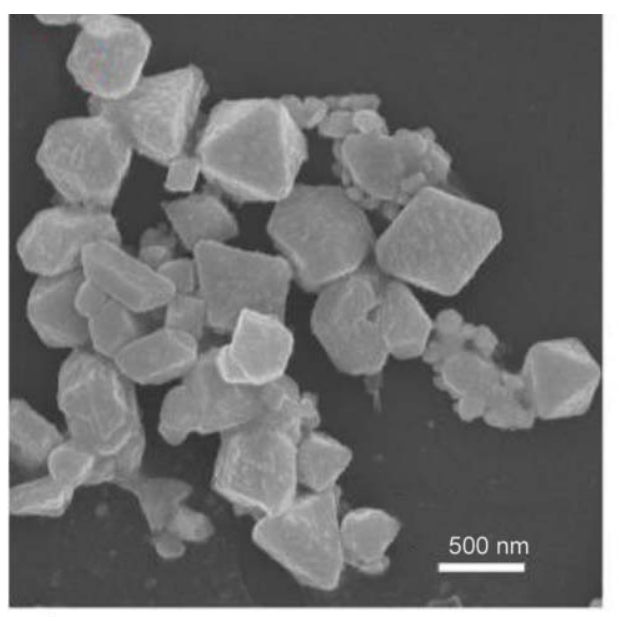

a)

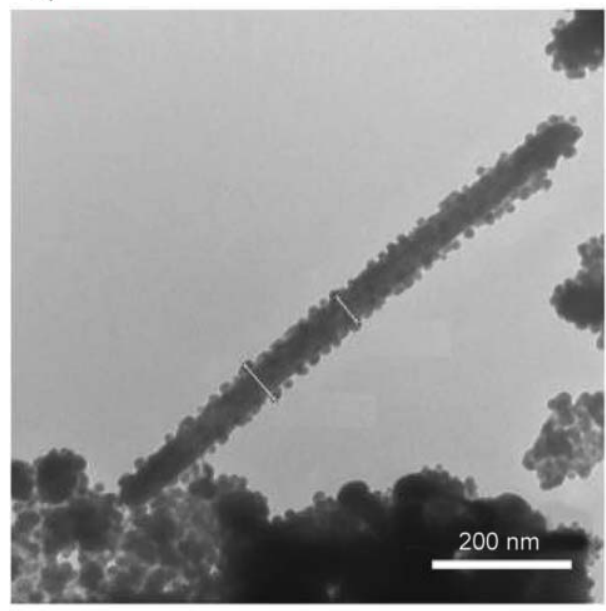

c) the system. This result could arise from the lack of reduction competition between the silver and iron cations. Reduction competition could be considered as another method to control the reaction rate. On the other hand, trapezoidal and triangular plate-like structures were found in Figure 7a, which demonstrated that they were used as seeds for the growth of the silver nanowires in DMF solution [45].

In the XRD analysis (Figure 8), all the products showed diffraction peaks from silver, as the (111), (200), (220), (222), and (311) FCC facets were clearly observed in the spectra. Nonetheless, as the concentration of iron chloride increased, silver chloride signals were observed in the XRD patterns.

Theoretically, to fabricate silver nanowires, the added silver cations must coordinate via polar sites and high electron density lone pairs to the highly ordered soft template in the system. The reduction of silver cations occurs easily at elevated temperatures. Accordingly, the predesigned heat-induced reversible $\beta-C D$ sol-gel template is an effective system for fabricating

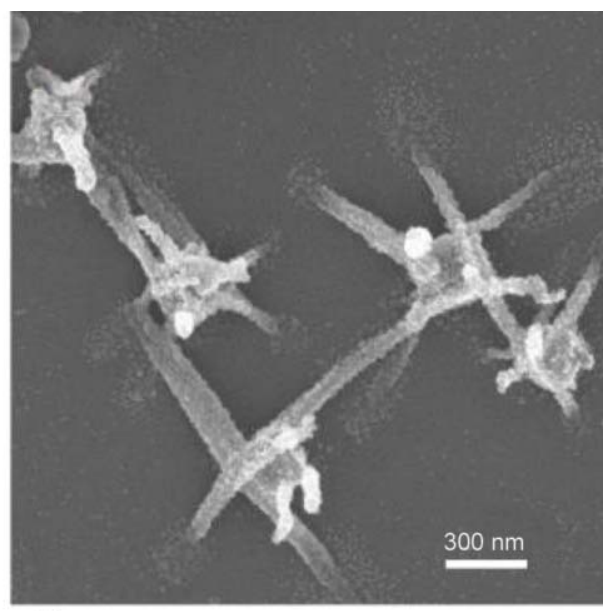

b)

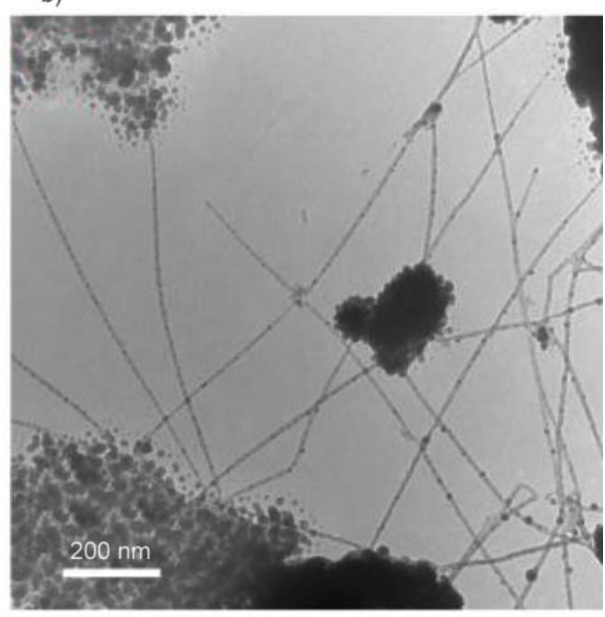

d)

Figure 7. (a, b) SEM and (c, d) TEM images of the three different products observed in (a) F1, (b) F4, (c) F3, (d) F4 with different iron chloride concentrations, showing the morphological evolution of the Ag nanostructures. 


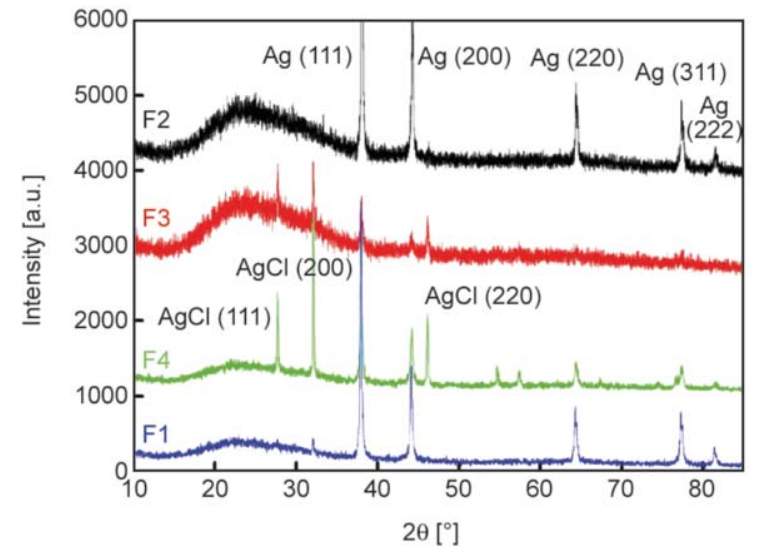

Figure 8. XRD analysis of the fabricated samples with different iron chloride concentrations.

silver nanowires. Moreover, the presence of iron chloride collapses the gel state, leading to a partial gel state. The partial gel state contains self-assembled formations in the solvent, which allows the ions to easily move and aggregate. With the assistance of lithium chloride and iron chloride, the silver cations were reduced at elevated temperatures.

The first nucleation point for silver nanowires is typically multiply twinned particles exhibiting nearfivefold symmetry. As shown in Figure 9, the most commonly observed polyhedral are decahedrons, and continuous nucleation occurs on the (111) facet for anisotropic growth in the [110] direction. When thin silver nanofibers are synthesized, they aggregate with other silver nanofibers, forming longer and wider nanowires. The ends of the nanowires could then be sometimes sensitized, attaching to other silver

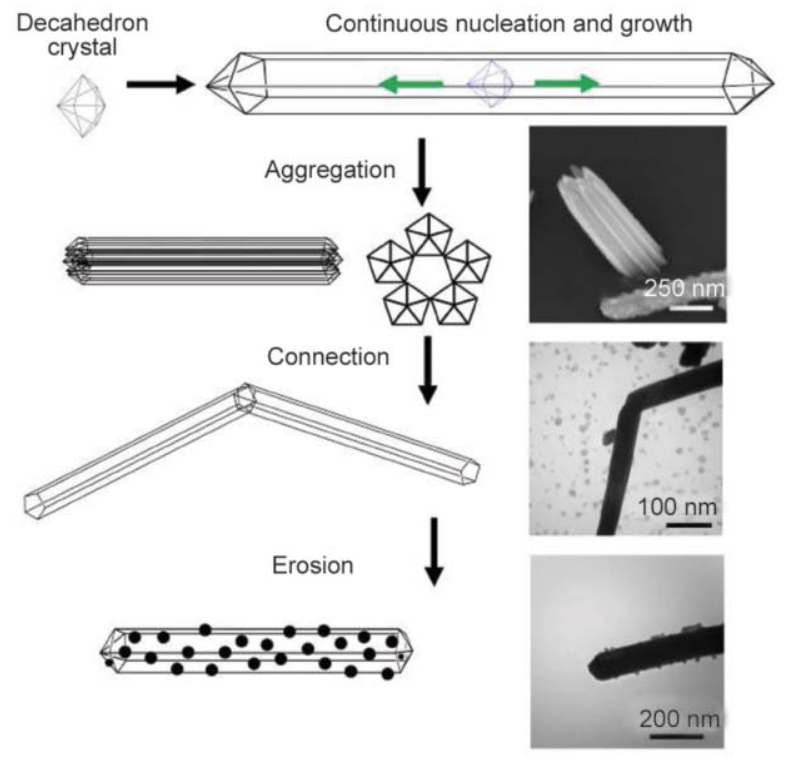

Figure 9. Schematic illustration of the growth of the sliver nanowires. nanowires to lengthen themselves. Figure 9 shows a bent connection of two growing silver nanowires. At the end of the synthesis, the silver nanowires can be etched by aqueous ammonia, and the most erosive region is the (100) facet [46].

\section{Conclusions}

In summary, silver nanowires were successfully fabricated via a sol-gel template using a heat-induced $\beta$-cyclodextrin organogel. The self-assembled highdensity $\beta$-cyclodextrin sol-gel template fabricated silver nanowires with long lengths. Studying the effect of the silver nitrate concentration showed that the lengths of the silver nanowires increased as the ratio of silver cations to chloride ions increased. The investigations of the effects of the iron chloride concentrations indicated that the presence of iron ions prevented etching of the silver materials.

\section{Acknowledgements}

The authors would like to thank the National Science Council (NSC) of the Republic of China (Taiwan) for financially supporting this research under Contract No.NSC104-2923-E006-004-MY3.

\section{References}

[1] Seiler H. G., Sigel H., Sigel A.: Handbook on toxicity of inorganic compounds. Marcel Dekker, New York (1988).

[2] Chen X., Schluesener H. J.: Nanosilver: A nanoproduct in medical application. Toxicology Letters, 176, 1-12 (2008). https://doi.org/10.1016/j.toxlet.2007.10.004

[3] Nowack B., Krug H. F., Height M.: 120 years of nanosilver history: Implications for bpolicy makers. Environmental Science and Technology, 45, 1177-1183 (2011). https://doi.org/10.1021/es103316q

[4] Hueso L., Mathur N.: Nanotechnology: Dreams of a hollow future. Nature, 427, 301-304 (2004). https://doi.org/10.1038/427301a

[5] Alivisatos A. P.: Perspectives on the physical chemistry of semiconductor nanocrystals. The Journal of Physical Chemistry, 100, 13226-13239 (1996). https://doi.org/10.1021/jp9535506

[6] Kamat P. V.: Photophysical, photochemical and photocatalytic aspects of metal nanoparticles. The Journal of Physical Chemistry B, 106, 7729-7744 (2002). https://doi.org/10.1021/jp0209289

[7] Ahmadi T. S., Wang Z. L., Green T. C., Henglein A., El-Sayed M. A.: Shape-controlled synthesis of colloidal platinum nanoparticles. Science, 272, 1924-1925 (1996). https://doi.org/10.1126/science.272.5270.1924 
[8] Wu Y., Xiang J., Yang C., Lu W., Lieber C. M.: Singlecrystal metallic nanowires and metal/semiconductor nanowire heterostructures. Nature, 430, 61-65 (2004). https://doi.org/10.1038/nature02674

[9] Sun Y., Xia Y.: Shape-controlled synthesis of gold and silver nanoparticles. Science, 298, 2176-2179 (2002). https://doi.org/10.1126/science.1077229

[10] Pinna N., Weiss K., Urban J., Pileni M-P.: Triangular CdS nanocrystals: Structural and optical studies. Advanced Materials, 13, 261-264 (2001).

https://doi.org/10.1002/1521-4095(200102)13:4<261::AIDADMA261>3.0.CO;2-X

[11] Bradley J. S., Tesche B., Busser W., Maase M., Reetz M. T.: Surface spectroscopic study of the stabilization mechanism for shape-selectively synthesized nanostructured transition metal colloids. Journal of the American Chemical Society, 122, 4631-4636 (2000). https://doi.org/10.1021/ja992409y

[12] Wang X., Zhuang J., Peng Q., Li Y.: A general strategy for nanocrystal synthesis. Nature, 437, 121-124 (2005). https://doi.org/10.1038/nature03968

[13] Duan X., Huang Y., Cui Y., Wang J., Lieber C. M.: Indium phosphide nanowires as building blocks for nanoscale electronic and optoelectronic devices. Nature, 409, 66-69 (2001). https://doi.org/10.1038/35051047

[14] Zhang J., Liu J., Wang S., Zhan P., Wang Z., Ming N.: Facile methods to coat polystyrene and silica colloids with metal. Advanced Functional Materials, 14, 10891096 (2004). https://doi.org/10.1002/adfm.200400119

[15] Yener D. O., Sindel J., Randall C. A., Adair J. H.: Synthesis of nanosized silver platelets in octylamine-water bilayer systems. Langmuir, 18, 8692-8699 (2002). https://doi.org/10.1021/la011229a

[16] Maillard M., Giorgio S., Pileni M. P.: Tuning the size of silver nanodisks with similar aspect ratios: Synthesis and optical properties. The Journal of Physical Chemistry B, 107, 2466-2470 (2003).

https://doi.org/10.1021/jp022357q

[17] Chen S., Carroll D. L.: Silver nanoplates: Size control in two dimensions and formation mechanisms. The Journal of Physical Chemistry B, 108, 5500-5506 (2004). https://doi.org/10.1021/jp031077n

[18] Washio I., Xiong Y., Yin Y., Xia Y.: Reduction by the end groups of poly(vinyl pyrrolidone): A new and versatile route to the kinetically controlled synthesis of $\mathrm{Ag}$ triangular nanoplates. Advanced Materials, 18, 1745 1749 (2006). https://doi.org/10.1002/adma.200600675

[19] Bastys V., Pastoriza-Santos I., Rodríguez-González B., Vaisnoras R., Liz-Marzán L. M.: Formation of silver nanoprisms with surface plasmons at communication wavelengths. Advanced Functional Materials, 16, 766 773 (2006).

https://doi.org/10.1002/adfm.200500667
[20] Deivaraj T. C., Lala N. L., Lee J. Y.: Solvent-induced shape evolution of PVP protected spherical silver nanoparticles into triangular nanoplates and nanorods. Journal of Colloid and Interface Science, 289, 402-409 (2005).

https://doi.org/10.1016/j.jcis.2005.03.076

[21] Zhang D., Qi L., Yang J., Ma J., Cheng H., Huang L.: Wet chemical synthesis of silver nanowire thin films at ambient temperature. Chemistry of Materials, 16, 872876 (2004).

https://doi.org/10.1021/cm0350737

[22] Tian M., Wang J., Kurtz J., Mallouk T. E., Chan M. H. W.: Electrochemical growth of single-crystal metal nanowires via a two-dimensional nucleation and growth mechanism. Nano Letters, 3, 919-923 (2003).

https://doi.org/10.1021/n1034217d

[23] Govindaraj A., Satishkumar B. C., Nath M., Rao C. N. R.: Metal nanowires and intercalated metal layers in single-walled carbon nanotube bundles. Chemistry Materials, 12, 202-205 (2000).

https://doi.org/10.1021/cm9905460

[24] Lu F-F., Yu H-Y., Zhou Y., Yao J-M.: Spherical and rodlike dialdehyde cellulose nanocrystals by sodium periodate oxidation: Optimization with double response surface model and templates for silver nanoparticles. Express Polymer Letters, 10, 965-976 (2016). https://doi.org/10.3144/expresspolymlett.2016.90

[25] Peponi L., Tercjak A., Martin L., Mondragon I., Kenny J. M.: Morphology-properties relationship on nanocomposite films based on poly(styrene-block-diene-blockstyrene) copolymers and silver nanoparticles. Express Polymer Letters, 5, 104-118 (2011). https://doi.org/10.3144/expresspolymlett.2011.12

[26] Braun E., Eichen Y., Sivan U., Ben-Yoseph G.: DNAtemplated assembly and electrode attachment of a conducting silver wire. Nature, 391, 775-778 (1998). https://doi.org/10.1038/35826

[27] Shumaila, Alam M., Siddiqui A. M., Husain M.: Synthesis, characterization and properties of Se nanowires intercalated polyaniline/Se nanocomposites. Express Polymer Letters, 7, 723-732 (2013).

https://doi.org/10.3144/expresspolymlett.2013.70

[28] Monerris M., Broglia M., Yslas I., Barbero C., Rivarola C.: Antibacterial polymeric nanocomposites synthesized by in-situ photoreduction of silver ions without additives inside biocompatible hydrogel matrices based on $N$-isopropylacrylamide and derivatives. Express Polymer Letters, 11, 946-962 (2017). https://doi.org/10.3144/expresspolymlett.2017.91

[29] Murphy C. J., Jana N. R.: Controlling the aspect ratio of inorganic nanorods and nanowires. Advanced Materials, 14, 80-82 (2002).

https://doi.org/10.1002/1521-4095(20020104)14:1<80::AIDADMA80>3.0.CO;2-\# 
[30] Gao P., Zhan C., Liu M.: Controlled synthesis of double- and multiwall silver nanotubes with template organogel from a bolaamphiphile. Langmuir, 22, 775 779 (2006).

https://doi.org/10.1021/la0517787

[31] Jin Q., Zhang L., Cao H., Wang T., Zhu X., Jiang J., Liu M.: Self-assembly of copper(II) ion-mediated nanotube and its supramolecular chiral catalytic behavior. Langmuir, 27, 13847-13853 (2011).

https://doi.org/10.1021/la203110z

[32] Majumder J., Deb J., Das M. R., Jana S. S., Dastidar P.: Designing a simple organic salt-based supramolecular topical gel capable of displaying in vivo self-delivery application. Chemical Communications, 50, 1671-1674 (2014). https://doi.org/10.1039/C3CC48513G

[33] Tao L., Huo Z., Dai S., Ding Y., Zhu J., Zhang C., Zhang B., Yao J., Nazeeruddin M. K., Grätzel M.: Stable quasi-solid-state dye-sensitized solar cells using novel low molecular mass organogelators and roomtemperature molten salts. The Journal of Physical Chemistry C, 118, 16718-16726 (2014).

https://doi.org/10.1021/jp412717y

[34] Morita C., Tanuma H., Kawai C., Ito Y., Imura Y., Kawai T.: Room-temperature synthesis of two-dimensional ultrathin gold nanowire parallel array with tunable spacing. Langmuir, 29, 1669-1675 (2013).

https://doi.org/10.1021/la304925e

[35] Nguyen N-T., Liu J-H.: Wet chemical synthesis of silver nanowires based on a soft template of cholesteryl pyridine carbamate organogel. Science of Advanced Materials, 7, 1282-1290 (2015).

https://doi.org/10.1166/sam.2015.2042

[36] Coleman A. W., Munoz M., Chatjigakis A. K., Cardot P.: Classification of the solubility behaviour of $\beta$-cyclodextrin in aqueous-CO-solvent mixtures. Journal of Physical Organic Chemistry, 6, 651-659 (1993).

https://doi.org/10.1002/poc.610061203

[37] He Y., Shen X., Chen Q., Gao H.: Characterization and mechanism study of micrometer-sized secondary assembly of $\beta$-cyclodextrin. Physical Chemistry Chemical Physics, 13, 447-452 (2011).

https://doi.org/10.1039/C0CP00899K
[38] Song L. X., Pan S. Z., Bai L., Dang Z., Du F. Y., Chen $J$.: Functional significance of molecule-ion interactions between a series of inorganic salts and $\beta$-cyclodextrin. Supramolecular Chemistry, 23, 447-454 (2011). https://doi.org/10.1080/10610278.2010.544737

[39] Izawa H., Kawakami K., Sumita M., Tateyama Y., Hill J. P., Ariga K.: $\beta$-cyclodextrin-crosslinked alginate gel for patient-controlled drug delivery systems: Regulation of host-guest interactions with mechanical stimuli. Journal of Materials Chemistry B, 1, 2155-2161 (2013). https://doi.org/10.1039/C3TB00503H

[40] Ionita G., Marinescu G., Ilie C., Anghel D. F., Smith D. K., Chechik V.: Sorption of metal ions by poly(ethylene glycol)/ $\beta$-CD hydrogels leads to gel-embedded metal nanoparticles. Langmuir, 29, 9173-9178 (2013). https://doi.org/10.1021/la401541p

[41] Li Z., Hao A., Hao J.: Formation of heat-triggered supramolecular organogel in which $\beta$-cyclodextrin as sole gelato. Colloids and Surfaces A: Physicochemical and Engineering Aspects, 441, 8-15 (2014). https://doi.org/10.1016/j.colsurfa.2013.08.078

[42] Wang Z., Liu J., Chen X., Wan J., Qian Y.: A simple hydrothermal route to large-scale synthesis of uniform silver nanowires. Chemistry A European Journal, 11, 160163 (2005).

https://doi.org/10.1002/chem.200400705

[43] Schuette W. M., Buhro W. E.: Silver chloride as a heterogeneous nucleant for the growth of silver nanowires. ACS Nano, 7, 3844-3853 (2013). https://doi.org/10.1021/nn400414h

[44] Wiley B., Sun Y., Xia Y.: Polyol synthesis of silver nanostructures: Control of product morphology with Fe(II) or Fe(III) species. Langmuir, 21, 8077-8080 (2005). https://doi.org/10.1021/la050887i

[45] Tsuji M., Tang X., Matsunaga M., Maeda Y., Watanabe M.: Shape evolution of flag types of silver nanostructures from nanorod seeds in PVP-assisted DMF solution. Crystal Growth Dessign, 10, 5238-5243 (2010). https://doi.org/10.1021/cg101041m

[46] Gao Y., Fu Y., Sun W., Sun Y., Wang H., Wang F., Zhao J.: Investigation on the mechanical behavior of fivefold twinned silver nanowires. Computational Materials Science, 55, 322-328 (2012). https://doi.org/10.1016/j.commatsci.2011.11.005 